\title{
Primeiro registro fóssil de Alligatoridae do Mato Grosso do Sul e o valor sistemático das rosáceas em Caimaninae
}

\author{
Caio Fabricio Cezar Geroto ${ }^{1}$ (), Alessandro Marques de Oliveira ${ }^{2}$ (1) \& Thais \\ Agrella Janolla ${ }^{1}$
}

(1) Universidade Paulista, Instituto de Ciências e Saúde, Ciências Biológicas, Laboratório de Ecologia Estrutural e Funcional de Ecossistemas, Avenida Independência 210, Éden, Sorocaba 18087-101, São Paulo, Brasil. E-mail: cgeroto@gmail.com, tha.janolla@gmail.com

(2) Universidade Federal do ABC, Laboratório de Sistemática e Diversidade, Avenida dos Estados 5001, Bangu, Santo André 09210-580, São Paulo, Brasil. E-mail: biolessandro@gmail.com

Geroto C.F.C., Oliveira A.M. \& Janolla T.A. (2019) Primeiro registro fóssil de Alligatoridae do Mato Grosso do Sul e o valor sistemático das rosáceas em Caimaninae. Pesquisa e Ensino em Ciências Exatas e da Natureza, 3(1): 98-115. http://dx.doi.org/10.29215/pecen.v3i1.1152

Editor acadêmico: Rodrigo Giesta Figueiredo. Recebido: 05 Junho 2018. Aceito: 10 Abril 2019. Publicado: 27 Maio 2019.

Resumo: No estado do Mato Grosso do Sul, fósseis de vertebrados Pleistocênicos ou Holocênicos são encontrados majoritariamente em grutas calcárias da Serra da Bodoquena. Além disso, na maioria das vezes os fósseis pertencem à mamíferos de grande porte, como por exemplo preguiças gigantes, tatus, gliptodontes, proboscídeos e grandes carnívoros. No entanto, as ocorrências de outros vertebrados fósseis são raras. Diante do exposto, este trabalho teve por objetivos realizar a primeira descrição de fósseis de Crocodylia provenientes do rio Formoso, próximo à foz com o rio Miranda, no município de Bonito-MS. Em adição, alguns comentários sobre sistemática foram realizados. Os elementos fósseis são dois osteodermos. Embora as características morfológicas dos osteodermos fósseis não permitam a identificação de sua posição precisa (dorsais ou caudais), os resultados das análises comparativas por meio da morfometria linear são sugestivos para identificação de provável Melanosuchus cf. niger, além de um espécime indeterminado de Eusuchia. Uma hipótese plausível seria considerar um possível caso de gigantismo em Caiman latirostris Daudin, 1802, cuja espécie ainda está presente na região. Portanto, os registros apresentados aqui ajudam a compor um quadro sobre a estrutura das comunidades de vertebrados durante o Quaternário Tardio de Mato Grosso do Sul.

Palavras chave: Osteodermo, Crocodylia, Rio Formoso, Quaternário, Mato Grosso do Sul.

First fossil record of Alligatoridae from Mato Grosso do Sul and the systematic value of rosacea in Caimaninae

Abstract: In the state of Mato Grosso do Sul, Pleistocene or Holocene vertebrate fossils are dicovered mostly in limestone caves from Serra da Bodoquena. In addition, such fossils commonly belong to large mammals, such as giant sloths, armadillos, glyptodonts, proboscids and large carnivores. However, the occurrence of other vertebrate fossils is scarce. In view of the above, the goal of this work was to perform the first description of Crocodylia fossils from the Formoso River, near the mouth of the Miranda River, in the municipality of Bonito-MS. In addition, some systematic comments were performed. The fossils studied herein are two osteoderms. Although the morphological characteristics of the osteoderms do not allow the identification of their precise position (dorsal or caudal), the results of the comparative analyses by means of linear morphometry are suggestive for the identification of Melanosuchus cf. niger, as well as an indeterminate specimen of Eusuchia. A plausible hypothesis would be to consider a putative case of gigantism in Caiman latirostris Daudin, 1802, whose species is still present in the region. Therefore, the fossils records presented here help us to compose a picture about the structure of the vertebrate communities during the Late Quaternary of Mato Grosso do Sul.

Key words: Osteoderm, Crocodylia, Formoso River, Quaternary, Mato Grosso do Sul. 


\section{Introdução}

A família Alligatoridae Gray, 1844 atualmente é representada pelos gêneros viventes Caiman Spix, 1825, Melanosuchus Gray, 1862, Paleosuchus Gray, 1862 e Alligator Daudin, 1809. Membros de tais gêneros ocorrem no leste dos Estados Unidos, América Central, América do Sul e leste da China. No Brasil são reconhecidas seis espécies pertencentes ao clado Caimaninae: Caiman crocodilus Linnaeus, 1758, Caiman latirostris Daudin, 1802, Caiman yacare Daudin, 1802, Melanosuchus niger Spix, 1825, Paleosuchus palpebrosus Cuvier, 1807 e Paleosuchus trigonatus Schneider, 1801 (Richardson et al. 2002; Vitt \& Caldwell 2009).

O registro fóssil de crocodilomorfos pleistocênicos na América do Sul (Figura 1) abrange táxons de Crocodylidae Cuvier, 1807, Gavialidae Hay, 1930 e Caimaninae, sendo mais abundante em território brasileiro (Fortier \& Rincón 2013; Cidade \& Hsiou 2018). Fósseis de Alligatoridae foram apontados para os estados de Rondônia (Andrade et al. 2010), Bahia (Fortier et al. 2010; Castro et al. 2014), Piauí (Guidon et al. 2009), Rio Grande do Norte (Porpino et al. 2004; Marinho et al. 2005), Ceará (Araújo-Júnior et al. 2010), Mato Grosso (Hirooka 2003), Rio Grande do Sul (Hsiou \& Fortier 2007) e Sergipe (de Melo França et al. 2014) e com certa dúvida Minas Gerais (Lydekker 1888; Patterson 1936).

No estado do Mato Grosso do Sul, a maioria dos fósseis de vertebrados provenientes de depósitos Quaternários são principalmente mamíferos (incluindo a megafauna pleistocênica), sendo mais raros a ocorrência de paleoherpetofauna (Salles et al. 2006; Scheffler et al. 2010; Pansani et al. 2016; Oliveira \& Cordeiro 2017; Oliveira et al. 2017). No entanto, durante obras do Gasoduto Bolívia/Brasil em 1995, foram coletados pelo processo de sucção de sedimentos do leito do rio Formoso (coordenadas aproximadas - Latitude $21.098654^{\circ}$ e Longitude $-56.228794^{\circ}$ próximo à foz com o rio Miranda, no município de Bonito, MS) restos de vertebrados bastante fragmentados, muitos dos quais foram previamente identificados como preguiças terrícolas e toxodontes (Oliveira 2013).

Dois osteodermos de crocodilomorfos procedentes da região da foz do Rio Formoso com o Rio Miranda foram mencionados por Oliveira (2013). No entanto, nenhum estudo comparativo, contendo uma descrição anatômica e sistemática destes fósseis foi realizada até o momento. Diante do exposto, o objetivo desta contribuição será apresentar uma descrição do material supramencionado com comentários sobre seu valor sistemático.

\section{Contexto geomorfológico}

O rio Formoso (Figura 2) está inserido no contexto da depressão do rio Miranda, onde encontra-se a bacia hidrográfica do Rio Miranda. Esta faz fronteira, ao norte, com a bacia do Rio Negro, a oeste, com a bacia do Rio Nabileque e, ao sul e a sudoeste, com a bacia do Rio Apa. A Noroeste, ela encontra um trecho da bacia do Rio Taquari. Este domínio geomorfológico/hidrográfico é composto por rochas terrígenas e carbonáticas (principalmente dolomíticas) dos grupos Corumbá e Cuiabá, deformadas da Faixa Paraguai. A oeste encontra-se o Planalto da Bodoquena e a leste o Planalto de Maracaju (Godoi 2001; Pereira et al. 2004). A depressão do rio Miranda forma depressões alagadiças, terraços elevados e secos durante boa parte do ano, e baixas colinas em terraços, leques aluviais de drenagem anastomosada (Godoi 2001). A cerca $47 \mathrm{~km}$, a sudoeste da foz entre os rios Formoso e Miranda, está a nascente do Formoso. Nesta mesma área tem sido registrado a ocorrência de fósseis da megafauna pleistocênica provenientes da gruta Nascente do Formoso (Salles et al. 2006).

Depósitos fossilíferos têm sido encontrados no leito dos rios Miranda e Formoso em pelo menos quatro localidades diferentes (Schefler et al. 2010; Oliveira 2013; Pansani et al. 2016). Os fósseis mais comuns são de vertebrados, principalmente mamíferos, além de conchas de gastrópodes e tufas calcárias. Geralmente as idades destes depósitos são atribuídas ao final do Pleistoceno ou Holoceno (Turcq et al. 1987; Boggiani et al. 2002; Filho et al. 2009; Scheffler et al. 2010; Pansani et al. 2016). 


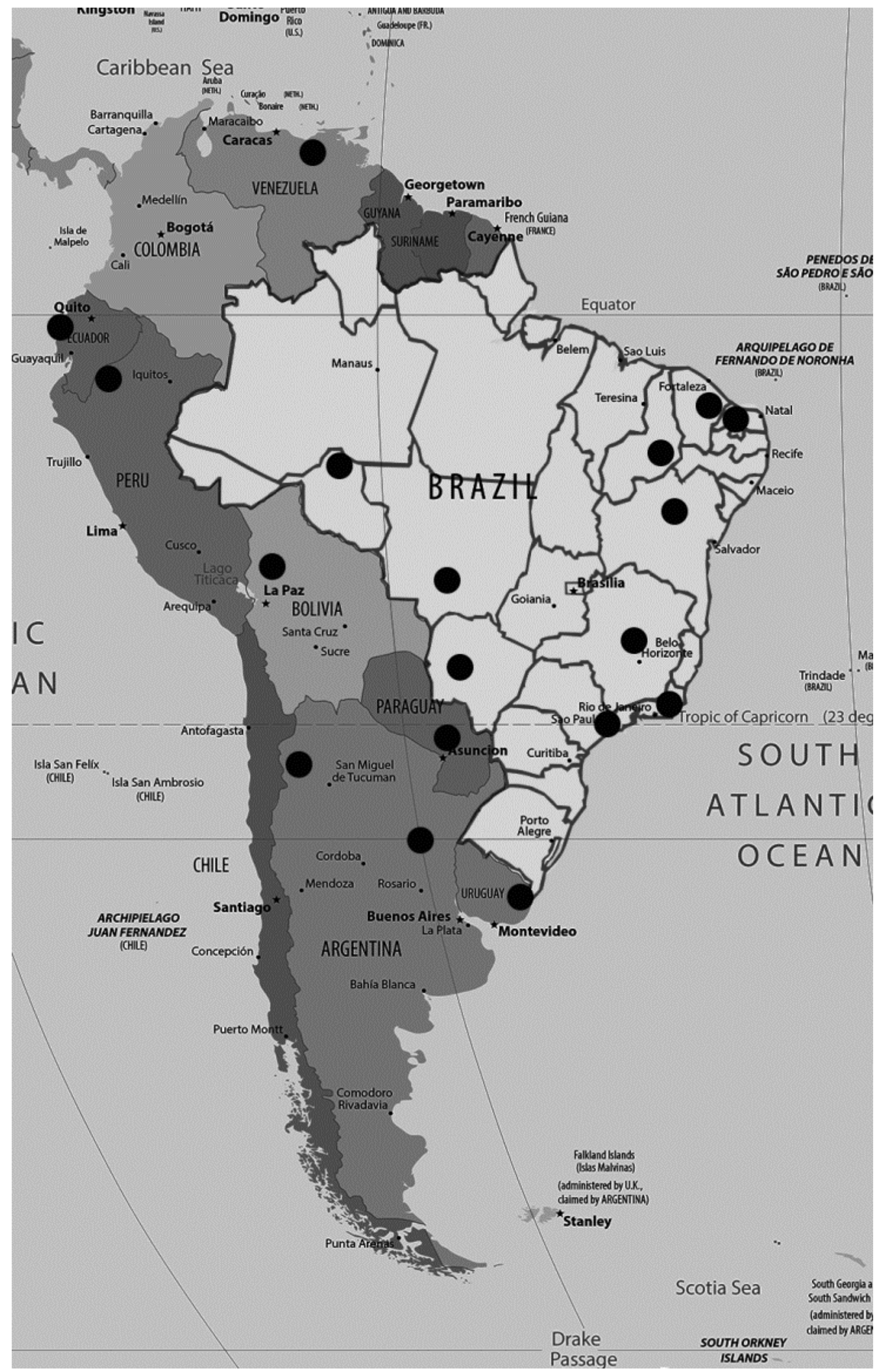

Figura 1. Mapa de ocorrências de fósseis de Alligatoridae na América do Sul. Imagem modificada de: Mapa político América do Sul (2019). 


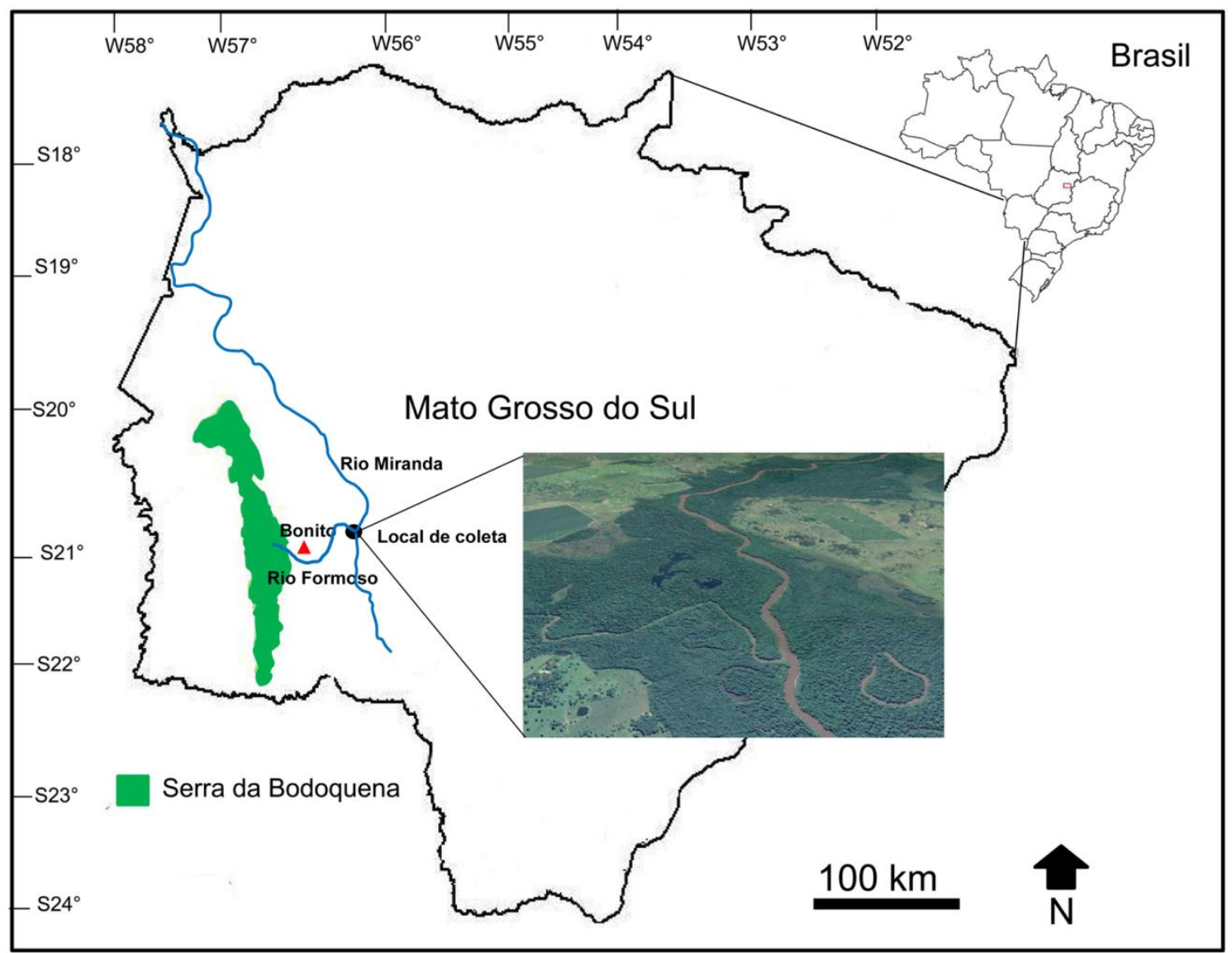

Figura 2. Local aproximado de coleta, na Foz do rio Formoso com o rio Miranda, Bonito (MS).

\section{Material e Métodos}

Os espécimes analisados (FOS-00054, FOS-00087) (Figura 3) encontram-se depositados na coleção de vertebrados fósseis (FOS) da Coleção Zoológica da Universidade Federal de Mato Grosso do Sul (ZUFMS), Campus Campo Grande. Para identificação e descrição do material seguiu-se Carvalho (1951), Romer (1997), Richardson et al. (2002) e Fortier \& Rincón (2013), além de observação pessoal de espécimes preservados em coleções.

A disponibilidade de osteodermos para comparação nas instituições é baixa, do mesmo modo, poucas coleções contam com espécimes de todos os táxons. Desse modo, o esforço de comparação exige a visita de instituições muito distantes. A dificuldade é maior especialmente com o Melanosuchus dada à facilidade com que os osteodermos se despreendem da pele após a morte do animal (Richardson et al. 2002). O processo, no entanto, pode ser evitado com a curtição do couro e preservação da pele (Richardson et al. 2002). Para a comparação com o material descrito no artigo observamos seis exemplares de pele de Caiman latirostris, seis de Caiman crocodilus, um de Melanosuchus niger, dois de Paleosuchus palpebrosus, três de Paleosuchus trigonatus e um da Gavialis gangeticus (Gmelin, 1789), depositados na coleção de Herpetologia do Museu de Zoologia da Universidade de São Paulo (MZUSP) e no Centro de Educação Ambiental do Parque Zoológico Municipal Quinzinho de Barros, em Sorocaba. Os números de tombo dos espécimes observados estão listados na Tabela 1. Também foi coletada informação de artigos disponíveis na literatura que trouxessem imagens e dados de espécimes que permitissem a comparação, como é o caso de Aligator mississipiensis (Daudin, 1802) (Tabela 1). Tal procedimento, embora não totalmente adequado, pois não permite a observação da ornamentação dos osteodermos, permite ao menos a comparação da morfologia e da presença de quilhas, bem como na determinação da posição anatômica.

Os trabalhos de coleta de dados por comparação revelaram um padrão emergente do arranjo dos osteodermos nucais dentro de Crocodylia (Tabela 2). Carvalho (1951) organizou os 
dados referentes aos Jacarea em termos de morfologia e variação de elementos nucais. Brochu (2013) apresentou um caráter relacionando o número de osteodermos nucais com os dorsais, mas poucos trabalhos tem se focado no uso da variação de osteodermos para identificação taxonômica em nível de espécies. Ao longo da verificação das coleções que tivemos acesso ficou clara a necessidade desse tipo de informação, visto que alguns espécimes estavam incorretamente identificados, mas tal equívoco teria sido facilmente resolvido se o número de osteodermos nucais tivesse sido levado em conta. Discutiremos esses casos abaixo à medida que surgirem.
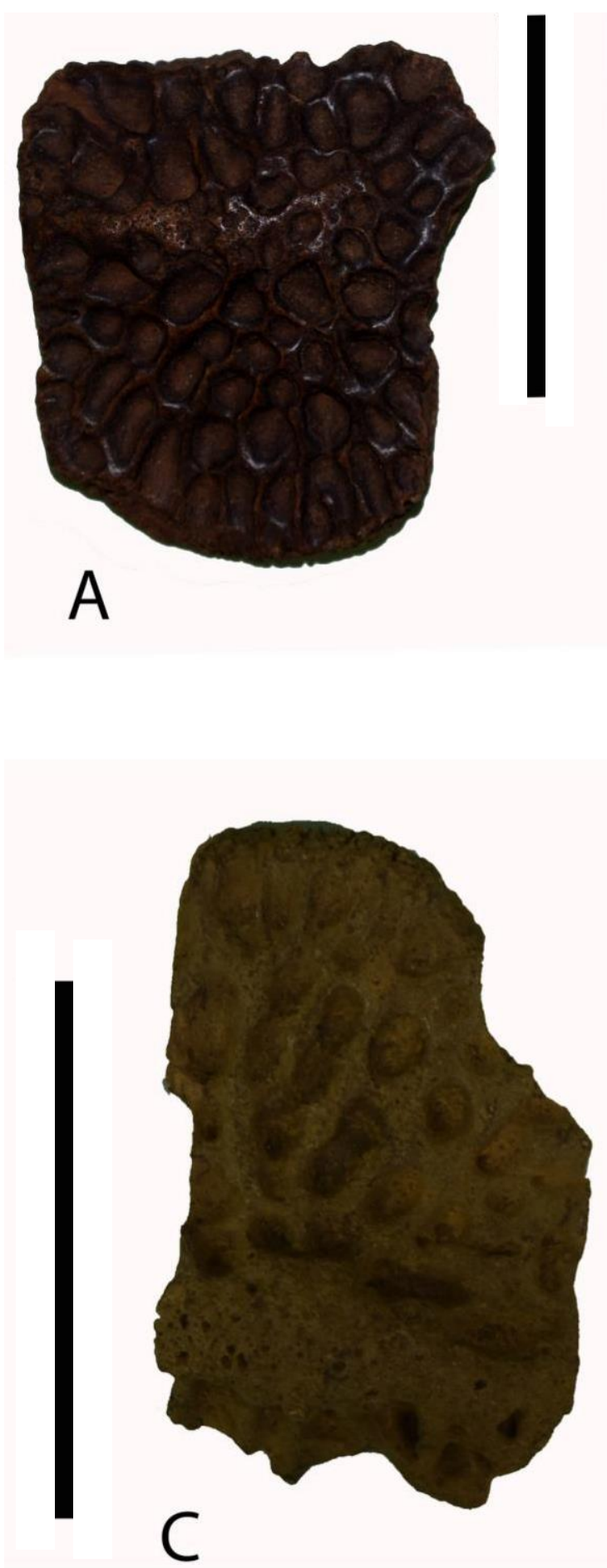
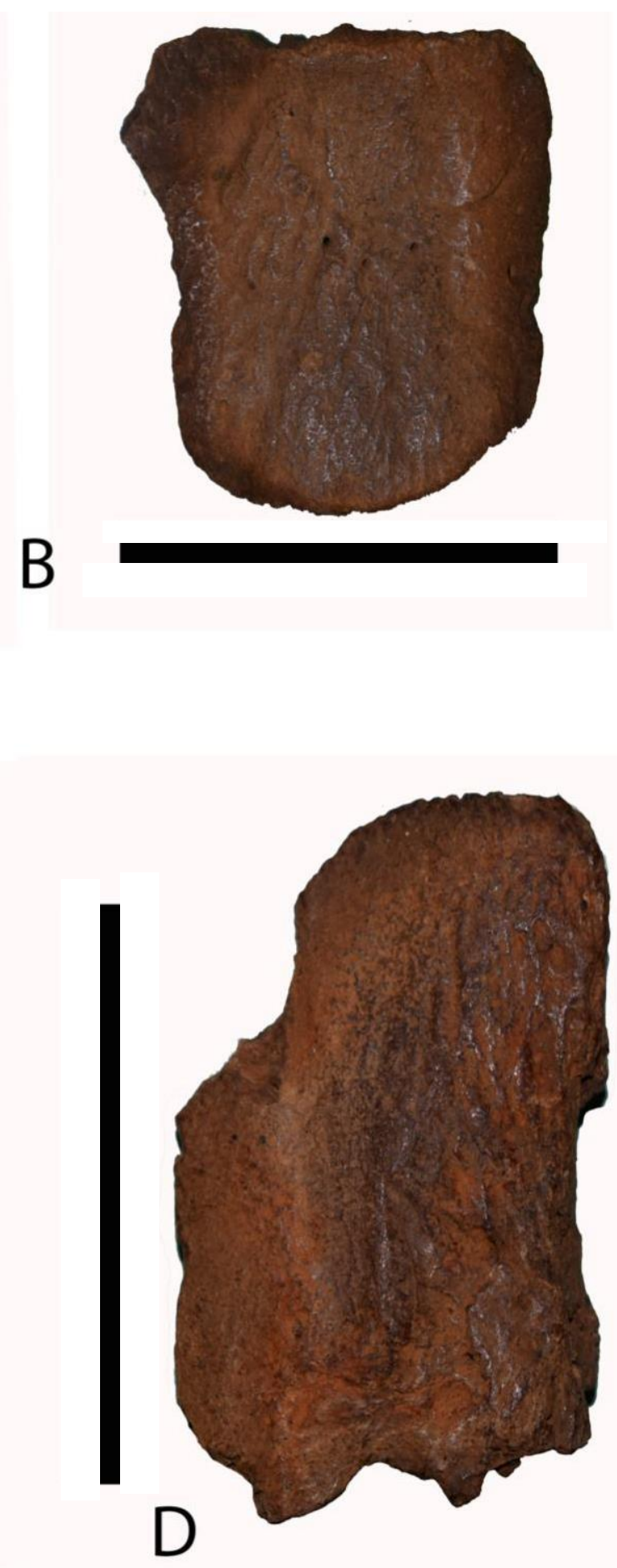

Figura 3. Osteodermos de Caimaninae. A-B) FOS-00054; C-D) FOS-00087. Escala 5 cm. 
Tabela 1. Espécimes observados em primeira mão para este estudo e o comprimento total de cada animal em milímetros.

\begin{tabular}{lc}
\hline Espécimes & Comprimento do animal (mm) \\
\hline Caiman latirostris & \\
MZUSP 2123 & 1630 \\
TRcro 01 & 1510 \\
TRcro 03 & 1430 \\
MZUSP2133 & 1200 \\
TRcro 04 & 900 \\
MZUSP 2132 & 780 \\
Caiman yacare & \\
2001 & 1270 \\
Caiman crocodilus & \\
MZUSP 2016 & 1620 \\
MZUSP 2017 & 1480 \\
TRcro 06 & 1210 \\
MZUSP 2018 & 1140 \\
TRcro 07 & 980 \\
Melanosuchus niger & 1700 \\
MZUSP 2157 & 1420 \\
FOS-00087 & 1010 \\
Alligator mississipiensis (Daudin, 1802) & 650 \\
DGH-AL-024 & 605 \\
Paleosuchus palpebrosus & \\
MZUSP 2172 & \\
MZUSP 2228 & \\
Paleosuchus trigonatus & \\
MZUSP 2190 & \\
MZUSP 2295 & \\
MZUSP 2294 & \\
Gavialis gangeticus & \\
MZUSP 2222 & \\
\hline & \\
\hline
\end{tabular}

Conforme discutido por Hill (2010) a nomenclatura para descrição de osteodermos apresenta uma série de problemas de terminologia que dificultam as comparações ou entendimento da posição ao longo do corpo dos crocodilomorfos. Seguiremos aqui a nomenclatura proposta por Hill (2010) onde a face mais externa é chamada de superficial, enquanto que a face do osteodermo que se volta para o corpo do animal é chamada de basal. Poços vão ser usados para se referir as concavidades profundas na ornamentação dos osteodermos, enquanto que cristas as superfícies ósseas entre os poços.

A posição dos osteodermos na rosácea nucal é considerada através de fileiras transversais aqui para propósito de discussão. Assim, quando um osteodermo específico for citado, ele será referido através de XTY, onde X indica a qual fileira transversal à qual ele pertence, iniciando-se da mais cranial para a mais caudal, e Y refere-se a qual é sua ordem da esquerda para direita. Assim, o osteodermos 1T2 trata-se do segundo osteodermo da primeira fileira transversal. Algumas espécies apresentam pequenos osteodermos ovais articulando-se lateralmente a área de contato entre duas fileiras e não fazendo parte diretamente da sequência 
de nenhuma delas, além de terem uma ocorrência extremamente variável dentro das espécies. Assim, optou-se aqui por denominá-los de osteodermos acessórios e não os contabilizar na contagem total dos osteodermos nucais.

A determinação do tamanho do animal ao qual FOS-00087 pertencia foi feita com base na comparação da proporção das medidas do osteodermo preservado, com aquelas obtidas dos espécimes analisados, seguindo um protocolo adaptado da metodologia de Tucker (1997), onde a área da superfície do osteodermo é correlacionada com o tamanho do animal. Para o cálculo da área, os osteodermos foram considerados como retângulos. Os valores de áreas dos osteodermos foram convertidos para medida de comparação em valores de log em base 10 e adicionados a uma matriz de dados morfométricos. A matriz foi analisada no programa Past 3 para componentes principais e análise cluster através do algoritmo Euclidiano. FOS-00087 e FOS00054 não foram incluídos nas análises por contarem com grande quantidade de missing data. As medidas de comprimento, largura, área e $\log 10$ usadas na análise cluster de todos os exemplares são listadas no material suplementar.

Lista de abreviações: FOS - Fóssil; MZUSP - Museu de Zoologia da Universidade de São Paulo; TRcro - Taxidermização Réptil crocodilia.

\title{
Resultados
}

\author{
Sistemática Paleontológica \\ Ordem Crocodylia Gmelin, 1789 \\ Família Alligatoridae Gray, 1844 \\ Subfamília Caimaninae Brochu, 1999 \\ Caimaninae indet.
}

Material estudado: FOS-00054, FOS-00087, osteodermo dorsal (Figura 3).

Localidade e horizonte: Serra da Bodoquena, Mato Grosso do Sul, Brasil.

Considerações: Possivelmente, o material possui idade entre o Pleistoceno Final e Holoceno, embora não haja datações absolutas para fósseis da região. Porém, a plausibilidade desta hipótese repousa sobre a identificação para o mesmo depósito de fósseis de preguiças gigantes e toxodontes (Oliveira et al. 2017), os quais são reconhecidos como mamíferos que teriam vivido até passagem Pleistoceno/Holoceno em diversas localidades do Brasil.

São poucos os osteodermos de espécies recentes em que a crista mediana das rosáceas divide a área da superfície do osteodermo em exatamente duas metades iguais. Tal distribuição simétrica foi observada em Crocodylidae, Gavialidae e Alligatoridae. Caimaninae apresenta uma assimetria na distribuição com a margem medial do osteodermo ocupando uma área de mais de $50 \%$, enquanto que a margem lateral corresponde a $40 \%$ da superfície do osteodermo. Uma vez que tafonomicamente as partes robustas e grandes possuem maior resistência ao retrabalhamento e transporte (Holz \& Simões 2002), interpretamos que a porção preservada de FOS-00087 equivale a sua margem medial. Desse modo, calculamos que o comprimento de 60.75 $\mathrm{mm}$ do osteodermo FOS-00087 deveria equivaler a aproximadamente 58\% da superfície, em comparação com a distribuição de área em superfície em outros espécimes. Isso levou nossos cálculos a propor um tamanho total de $106.47 \pm 29 \mathrm{~mm}$ para o osteodermo como um todo. Quando comparado à proporção do tamanho dos animais com seus respectivos osteodermos, chegamos a um provável tamanho mínimo de $5.00 \pm 0.32$ metros.

Embora classificado como Caiman crocodilus, MZUSP 2001 apresenta quatro fileiras transversais com dois osteodermos cada uma, possuindo dois osteodermos mais longos que largos, com crista central pouco proeminente e margens arredondadas e com dois osteodermos acessórios menores ao lado da segunda fileira transversal. Isso o difere dos demais Caiman 
crocodilus observados e o aproxima da descrição dos dados contidos na literatura para a espécie Caiman yacare (e.g., Carvalho 1951).

As análises cluster e multivariadas (Figuras 4-5) trouxeram resultados semelhantes entre si. Os espécimes de Paleosuchus e Caiman crocodilus, Caiman yacare e Melanosuchus niger mostraram-se morfologicamente próximos, com o principal fator de diferenciação sendo a área dos osteodermos da terceira e quarta fileira transversal. A proximidade entre Caiman crocodilus e Melanosuchus niger ocorreu pelos osteodermos da quinta fileira transversal. A morfologia do quarto osteodermo da primeira fileira transversal foi o fator que isolou Caiman latirostris dos demais.

Tabela 2. Espécies de Crocodylia com o número de fileiras transversais nucais e a distribuição dos osteodermos nas fileiras. Valores entre parênteses representam os osteodermos acessórios. Fil. tran. = Fileiras transversais; Ost. fil. = Osteodermos por fileiras.

\begin{tabular}{|c|c|c|c|}
\hline & Fil. tran. & Ost. fil. & Referências \\
\hline Caiman latirostris & 2 & 4,2 & Observação pessoal; Carvalho (1951) \\
\hline Caiman yacare & 5 & $\begin{array}{l}4,2(4), 2(4), 2 \text {, } \\
2\end{array}$ & Observação pessoal; Carvalho (1951) \\
\hline Caiman crocodilus & 5 & $4,2,2,2,2$ & Carvalho (1951); Clarac et al. (2018) \\
\hline Melanosuschus niger & 4 & $4,4,2,2$ & Observação pessoal; Carvalho (1951) \\
\hline Paleosuchus trigonatus & 3 & $2,2,2$ & $\begin{array}{l}\text { Observação pessoal; Carvalho (1951); } \\
\text { Magnusson (1992) }\end{array}$ \\
\hline Paleosuchus palpebrosus & 5 & $2,2,2,2,2$ & Observação pessoal; Carvalho (1951) \\
\hline Alligator mississipiensis & 2 & 2,2 & $\begin{array}{l}\text { Dubansky (2012); Chen et al. (2014); } \\
\text { Dubansky \& Dubansky (2018) }\end{array}$ \\
\hline Alligator sinensis Fauvel, 1879 & 3 & $2,2,2$ & Chen et al. (2014) \\
\hline Gavialis gangeticus & 2 & 2,2 & Observação pessoal \\
\hline Osteolemus tetraspis (Cope, 1861) & 2 & 2,2 & Wermuth \& Fuchs (1978) \\
\hline Tomistoma sp. & 3 & $2,2,2$ & Wermuth \& Fuchs (1978) \\
\hline $\begin{array}{l}\text { Crocodylus porosus (Schneider, } \\
1801 \text { ) }\end{array}$ & $2(1)$ & $2,2(2)$ & Wermuth \& Fuchs (1978) \\
\hline Crocodylus niloticus Laurenti, 1768 & 2 & 4,2 & Wermuth \& Fuchs (1978) \\
\hline Crocodylus acutus (Cuvier, 1807) & 2 & 2,2 & Wermuth \& Fuchs (1978) \\
\hline Crocodylus johnsoni Krefft, 1873 & 1 & 4 & Wermuth \& Fuchs (1978) \\
\hline $\begin{array}{l}\text { Crocodylus intermedius Graves, } \\
1819\end{array}$ & 2 & 2,2 & Wermuth \& Fuchs (1978) \\
\hline $\begin{array}{l}\text { Crocodylus moroletti (A.H.A. } \\
\text { Duméril \& Bibron, 1851) }\end{array}$ & $2(1)$ & $2,2(2)$ & Wermuth \& Fuchs (1978) \\
\hline $\begin{array}{l}\text { Crocodylus novaguinaea Schmidt, } \\
1928\end{array}$ & 2 & 2,2 & Wermuth \& Fuchs (1978) \\
\hline Crocodylus rhombifer Cuvier, 1807 & 2 & 4,2 & Wermuth \& Fuchs (1978) \\
\hline $\begin{array}{l}\text { Crocodylus siamensis (Schneider, } \\
\text { 1801) }\end{array}$ & $2(1)$ & $2,2,(2)$ & Wermuth \& Fuchs (1978) \\
\hline $\begin{array}{l}\text { Mecistops cataphractus (Cuvier, } \\
1825)\end{array}$ & 3 & $2,2,2$ & Wermuth \& Fuchs (1978) \\
\hline
\end{tabular}


Primeiro fóssil de Alligatoridae do MS

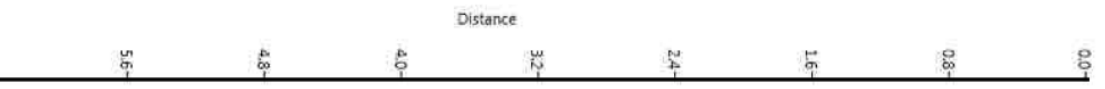

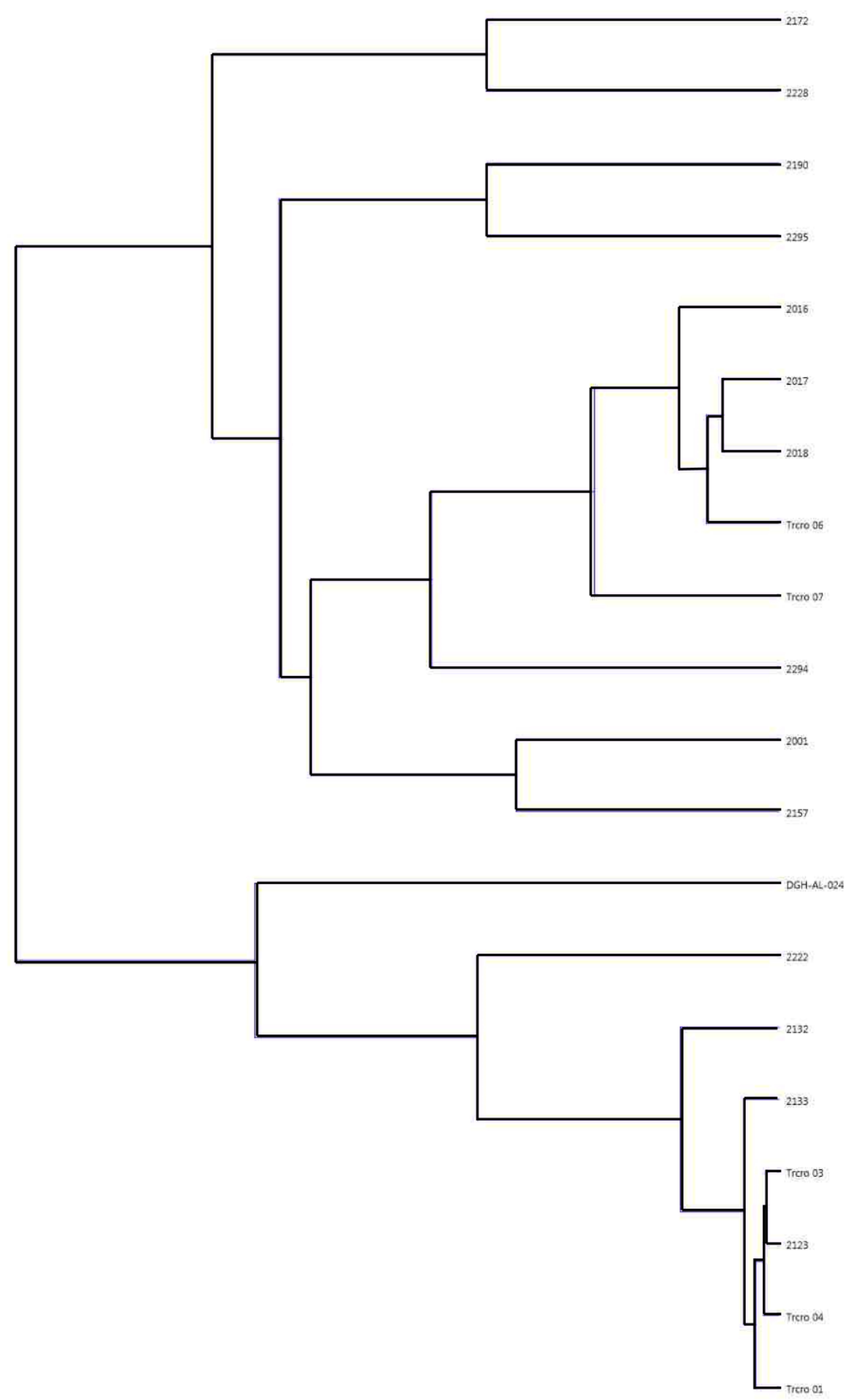

Figura 4. Análise cluster pelo algoritmo euclidiano. A análise retornou um coeficiente de correlação de 0.9426 . 


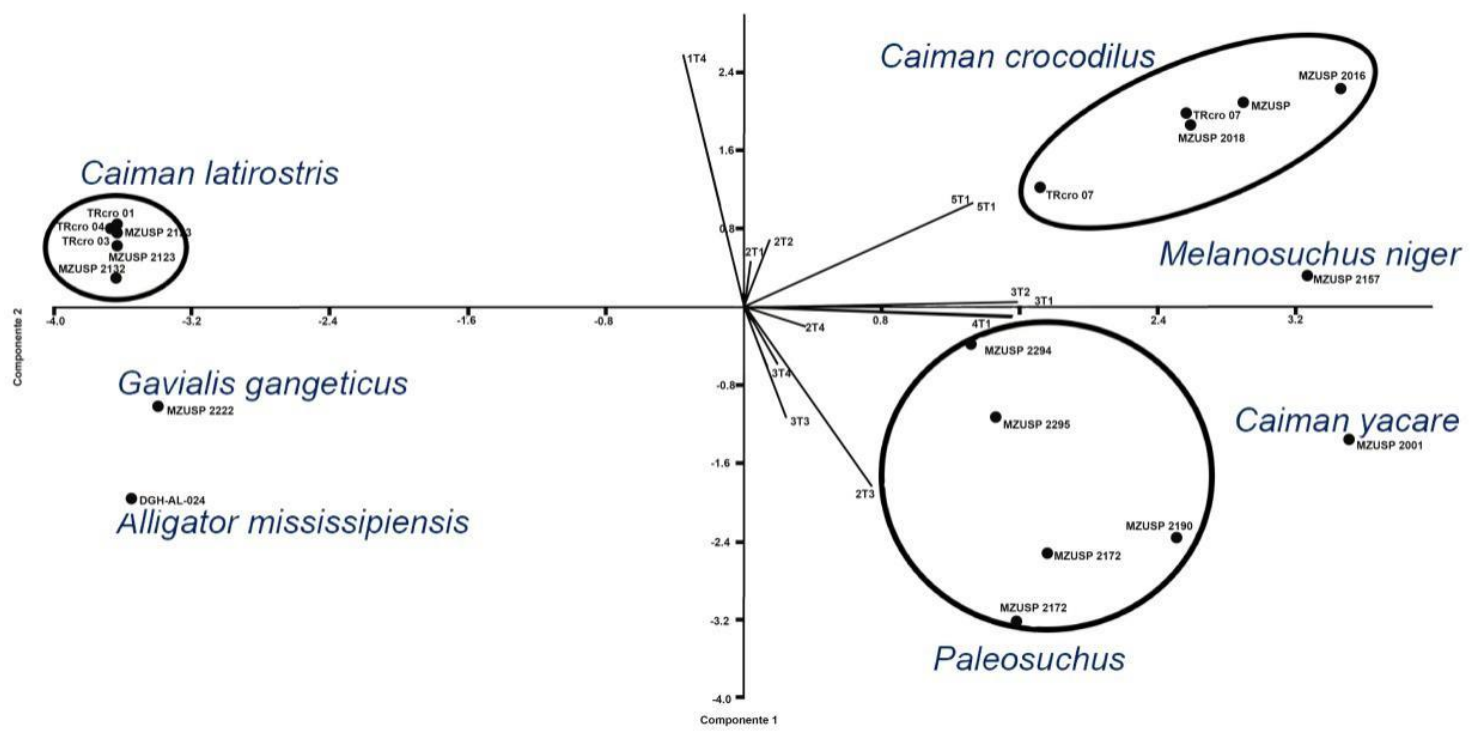

Figura 5. Análise multivariada mostrando a separação morfológica pela superfície dos osteodermos.

\section{Discussão}

Nenhum dos osteodermos fósseis compartilha características morfológicas que os permitam ser identificados como osteodermos dorsais ou caudais. Observamos que, de modo geral, em Jacarea, osteodermos dorsais são quadrados, quase retangulares, com razão comprimento largura próximo de 1 . Essa razão diminui à medida que se avança nas fileiras laterais quando os osteodermos se tornam mais compridos do que largos, com bordas arredondadas. À medida que se aproxima da porção mais distal da cauda, o número de osteodermos caudais diminui, seu tamanho aumenta e a quilha se torna mais pronunciada.

A morfologia dos elementos se assemelha às placas dérmicas dorsais e laterais de caimaníneos viventes (Romer 1997; Richardson et al. 2002; Fortier \& Rincón 2013), com formato retangular ou oval assimétrico e cristas separando as fossas profundas. A crista central de FOS00087, apesar de desgastada se mostra robusta e baixa, similar às cristas finas e altas de Caimaninae, Alligatorinae e Crocodylidae (Wermunt \& Fuchs 1978). Em Caiman latirostris, a razão próxima de 1 e decrescente se mantém, mas os osteodermos apresentam bordas arredondadas, sendo mais ovais. FOS-00054 também possui uma razão comprimento largura próxima de 1, no entanto, em FOS-00054 uma quilha bem desenvolvida está ausente, sendo mais provável que ele se trate de um elemento lateral do tronco. O osteodermo FOS-00087 mostra semelhanças morfológicas das quilhas, cristas e fossas com aqueles descritos por Fortier \& Rincón (2013) atribuídos a um indivíduo juvenil. É possível que as fossas mais largas e profundas, bem como as cristas mais finas de FOS-00054 possam indicar um indivíduo mais maduro, porém, não existe ainda um consenso sobre a relação entre a ornamentação óssea e a ontogenia (Buffrénil 1982).

Tucker (1997) encontrou uma forte correlação entre a proporção dos osteodermos e o tamanho de Crocodylus johnstoni. Desse modo, os osteodermos nucais podem ser usados como uma ferramenta para se determinar o tamanho de espécies extintas. Partindo desse resultado, o grande tamanho de FOS-00087 pode ser sugestivo para identificação do gênero Melanosuchus. O espécime estudado de Melanosuchus niger tratava-se provavelmente de um subadulto com 1400 mm de comprimento (1.40 metros). Os osteodermos que melhor se comparam ao material fóssil aqui descrito são aqueles da terceira transversal (3T2), com a largura maior que o comprimento (29.86 mm de largura e $24.57 \mathrm{~mm}$ de comprimento). Em um cálculo de proporções, um Melanosuchus niger adulto de $5000 \mathrm{~mm}$ (5 metros) teria osteodermos da terceira transversal com aproximadamente $105.10 \mathrm{~mm}$ de largura por $86.51 \mathrm{~mm}$ de comprimento. Desse modo, é 
possível que o material FOS-00087 possa ser classificado como Melanosuchus cf. niger. Outra possibilidade seria um caso de gigantismo num indivíduo de Caiman latirostris, espécie que ocorre atualmente na região. Purusaurus brasiliensis é um exemplo de caso de gigantismo dentro de Caimaninae, no entanto, não existem ocorrências de seus osteodermos descritos na literatura.

Ainda assim, se faz necessária uma comparação morfológica com os osteodermos das espécies atuais. Iniciaremos a partir das menos prováveis. O gênero Paleosuchus (Figura 6) apresenta uma variação no número de fileiras transversais, embora todas possuam apenas dois osteodermos. A espécie $P$. trigonatus apresenta três fileiras, enquanto que $P$. palpebrosus apresenta cinco. $\mathrm{O}$ mesmo ocorre com as espécies de Alligator, A. mississipiensis possui duas fileiras transversais de osteodermos, enquanto que $A$. sinensis possui três fileiras. Paleosuchus possui cristas centrais altas e longas craniocaudalmente, inclinando-se lateralmente. Essas cristas afinam-se em relação ao topo, diferente da crista de FOS-00087, larga, robusta e baixa, além de muito maior que os osteodermos de Paleosuchus. FOS-00087 assemelha-se morfologicamente aquele de Alligator mississipiensis conforme representado por Dubansky (2012) e Dubansky \& Dubansky (2018). No entanto, em Alligator, as cristas que separam os poços são altas e finas, enquanto os poços são irregulares (Chen et al. 2014), contrastando com FOS-00087 que apresenta poços circulares e cristas largas e baixas.

Caiman latirostris possui uma rosácea com duas fileiras transversais de osteodermos dorsais com quatro elementos na primeira fileira e dois na segunda (Figura 7). As mediais possuem duas linhas de osteodermos, enquanto que as laterais possuem apenas um osteodermo cada. Os osteodermos dorsais se distribuem em quatro fileiras com os mediais sendo mais retangulares e os laterais mais ovais. Comparativamente, os materiais fósseis são aproximadamente $144 \%$ maiores que os osteodermos de um Caiman latirostris recente, no entanto, $C$. latirostris só possui duas fileiras transversais de osteodermos com morfologia diferente daquela de FOS-00087. Caiman latirostris apresenta osteodermos mais arredondados, quase ovais, com quilhas deslocadas lateralmente em semelhança ao que ocorre em FOS-00054.

Caiman yacare apresenta cinco a quatro fileiras transversais na rosácea, seguindo a mesma distribuição que $C$. crocodilus, podendo ocorrer com dois pares de elementos acessórios, entre a segunda, terceira e quarta fileira (Figura 8). Variações na presença da última fileira da rosácea podem ser compreendidas como variações populacionais e só podem ser entendidos como variações de subespécie se ocorrem em mais de $75 \%$ dos indivíduos, conforme os resultados de Busack \& Pandya (2001). Elementos acessórios em rosáceas também ocorrem em Crocodylus porosus, C. moroletti e C. siamensis.

Caiman crocodilus apresenta uma rosácea com cinco fileiras transversais possuindo quatro elementos na primeira fileira e dois elementos na segunda, terceira, quarta e quinta fileiras (Figura 8). Também ocorrem osteodermos acessórios entre a segunda e terceira. Estes elementos acessórios não acompanham a fila dos osteodermos, estando em uma posição intermediaria. FOS-00087 é cerca de $83 \%$ maior que um osteodermo da terceira transversal de um C. crocodilus.

Em termos de número e morfologia das rosáceas variam pouco dentro do gênero, porém, apresentam uma variação maior entre os gêneros. Isso oferece uma segurança no momento de se usar as características dos osteodermos nucais, bem como seu número e distribuição nas rosáceas para a sistemática e taxonomia de espécies atuais e fósseis. Um padrão que parece ter emergido é que o clado Jacarea sensu Brochu (1997) possui como característica a presença de quatro osteodermos na primeira fileira da rosácea, podendo variar o padrão das fileiras posteriores entre quatro e dois osteodermos. Do mesmo modo, dentro de Crocodylidae (e.g., Crocodylus, Tomistoma e Mecistops), o padrão são dois osteodermos na primeira fileira transversal, com as únicas variações sendo $C$. niloticus, $C$. rhombifer e $C$. johnstoni com uma fileira transversal de quatro osteodermos. Nesse sentido, a primeira fileira transversal de osteodermos parece ser um caráter mais estável da rosácea. 

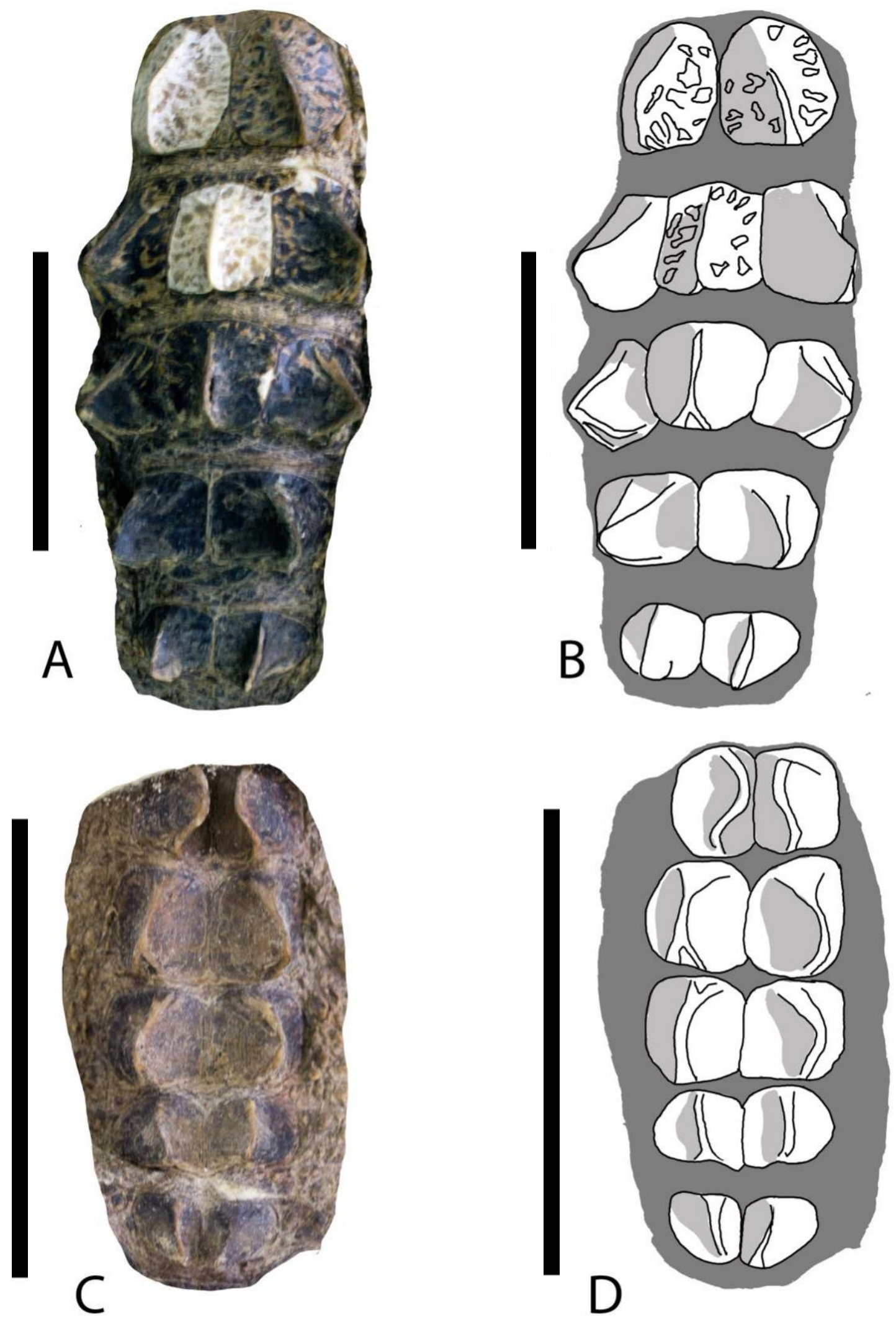

Figura 6. Variação das rosáceas no gênero Paleosuchus: A-B. Paleosuchus palpebrosus (MZUSP 2228); C-D. Paleosuchus trigonatus (MZUSP 2294). Barras de escala $=5 \mathrm{~cm}$. 

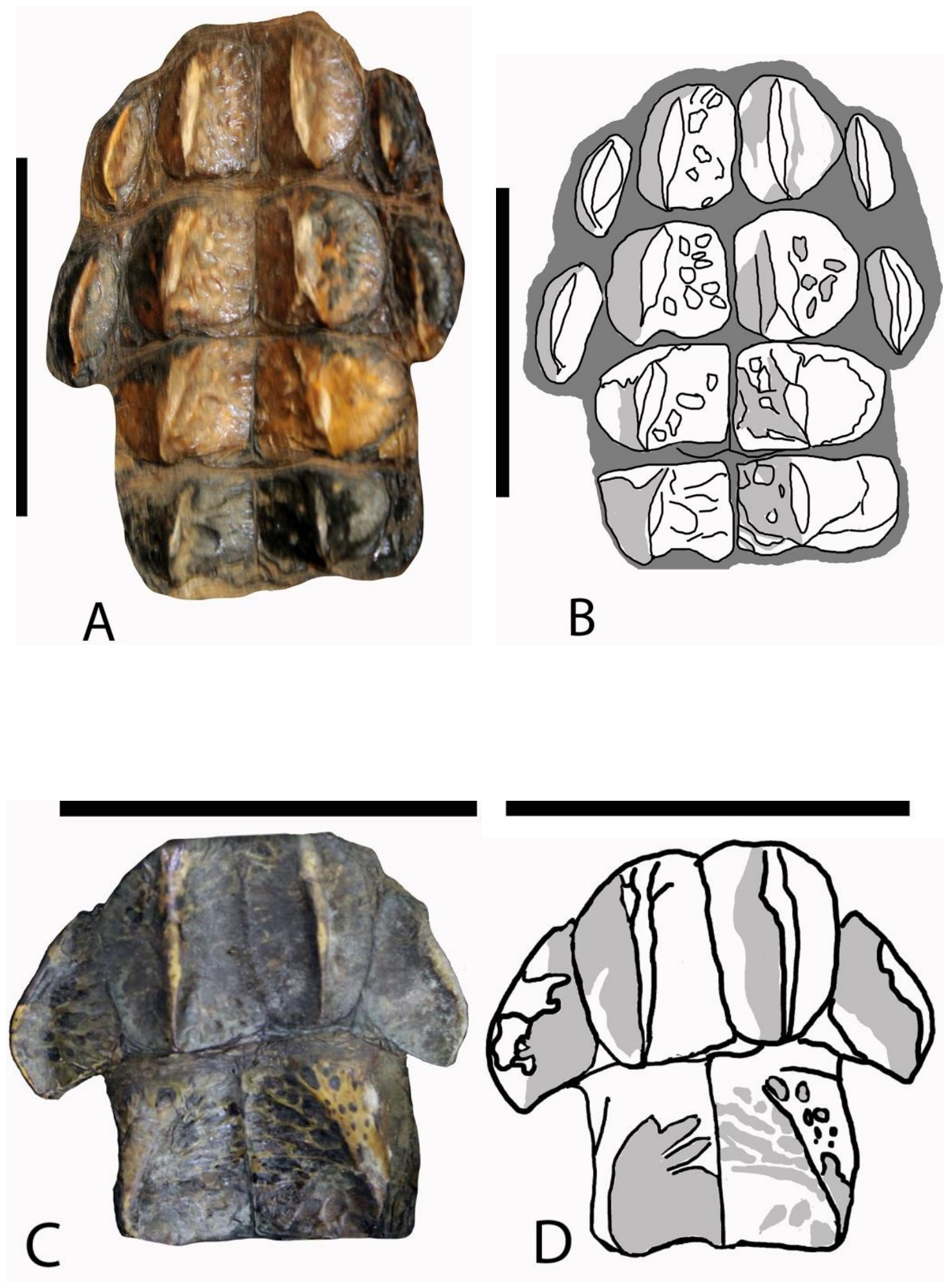

Figura 7. Rosáceas de Caimaninae: A-B. Melanosuchus niger (MZUSP 1420); C-D. Caiman latirostris (MZUSP 2133). Barras de escala $=5 \mathrm{~cm}$. 

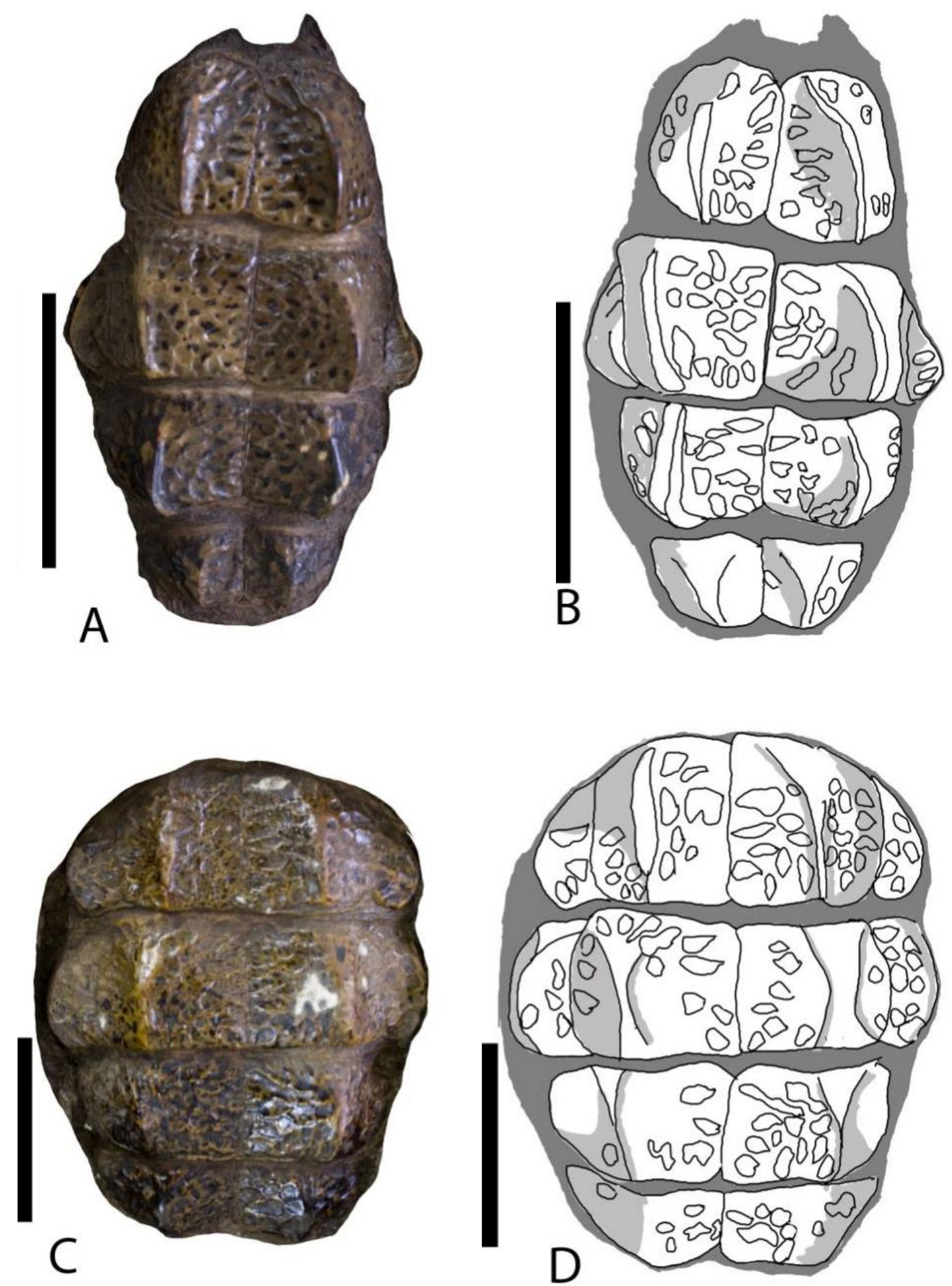

Figura 8. Variação das rosáceas entre as espécies: A-B. Caiman yacare (MZUSP 2001); G-D. Caiman crocodilus (MZUSP 2107). Barras de escala $=5 \mathrm{~cm}$.

De importância para sistemática, o padrão geral de morfologia e disposição dos osteodermos mostrou-se significativo. Poucos foram os casos de variação de número dos elementos e, ainda assim apenas abaixo da primeira fileira transversal, nesses casos ainda se manteve o padrão geral de morfologia. A presença de três ou mais fileiras transversais de osteodermos foi observada apenas em Alligatoridae e Tomistoma, com os casos extremos, 
quatro ou cinco ocorrendo dentro de Caimaninae. Crocodilydae e Gavialidae sempre apresentaram apenas dois osteodermos nucais nas rosáceas. Portanto, o número de fileiras transversais parece ser um caráter válido sistematicamente. Se esse for o caso, ele aproximaria Gavialidae de Crocodylidae e Caiman crocodilus e C. yacare de Melanosuchus niger.

Atualmente, o papel dos osteodermos tem sido repensado de um elemento primariamente de defesa para uma característica associada a movimento e inserção de musculatura (Salisbury \& Frey 2001; Salisbury et al. 2006). Nesse sentido, o número de fileiras transversais e de osteodermos nessas fileiras pode estar associado ao hábito de vida desses animais, especialmente, movimentação do pescoço durante a caça.

\section{Conclusões}

Existe um padrão visível de morfologia, comprovado pela morfometria linear, entre o arranjo dos osteodermos nucais de Alligatoridae. Este arranjo se aproxima em partes das propostas filogenéticas mais atuais para o grupo, mas apresenta algumas diferenças com relação a posição de Melanosuchus niger em relação a Caiman latirostris. FOS-00087 pertenceu a um Caimaninae de grande tamanho, podendo ser identificado com certa segurança morfológica como um osteodermos nucal da terceira transversal. Embora sua morfologia se aproxime daquela de Melanosuchus niger não foi possível afirmar com segurança essa identificação. FOS00054 é um osteodermo lateral padrão de Eusuchia Huxley, 1875. Não foi possível confirmar a sua identificação além disso.

\section{Agradecimentos}

Os autores agradecem a Peônia Brito de Moraes Pereira (Zoológico Municipal de Sorocaba), Aline Staskowian Benetti (Museu de Zoologica da USP) e Lauren Vonnahme (American Museum of Natural History) por disponibilizarem os exemplares e imagens sem os quais esse trabalho não seria possível. Agradecemos ao técnico Francisco Severo da Costa Neto (Coleção Zoológica da Universidade Federal de Mato Grosso do Sul - ZUFMS) pelo acesso aos fósseis de osteodermos. Também agradecemos aos editores Silvio Felipe e Rodrigo Giesta Figueiredo pela ajuda e oportunidade de publicação. Por fim, aos revisores anônimos cujas sugestões e críticas contribuíram para o enriquecimento do manuscrito.

\section{Referências}

Andrade L.C., Nascimento E.R., Tizuka M.M. \& Kipnis R. (2010) Primeira ocorrência da família Alligatoridae (Crocodilya) em depósitos fluviais do Rio Madeira, Porto Velho, RO (p. 72-82). In: da Silva R.C. \& Avilla L.S. (Orgs). VII Simpósio Brasileiro de Paleontologia de Vertebrados, 72. Rio de Janeiro: SBP.

Araújo-Júnior H.I., Pinheiro A.E.P., Ximenes C.L., Santos A.S.T. \& Porpino K.O. (2010) Primeiro registro de Caimaninae em depósitos pleistocênicos de tanques no Nordeste brasileiro (p. 1314). In: Vasconcellos F.M. (Org.). Jornada Fluminense de Paleontologia. Volume 5. Rio de Janeiro: UFRJ.

Boggiani P.C., Coimbra A.M., Gesicki A.L.D., Sial A.N., Ferreira V.P., Brenha-Ribeiro F. \& Flexor J.M. (2002) Tufas Calcárias da Serra da Bodoquena, MS: cachoeiras petrificadas ao longo dos rios (p. 249-259). In: Schobbenhaus C., Campos D.A., Queiroz E.T., Winge M. \& Berbet-Born M. (Eds). Sítios Geológicos e Paleontológicos do Brasil. Brasília: DNPM.

Brochu C. A. (1997) Morphology, fossil, divergence timing and Phylogenetic relationships of Gavialis. Systematic Biology, 46(3): 479-522. DOI: 10.1093/sysbio/46.3.479

Brochu C.A. (1999) Phylogenetics, Taxonomy, and Historical Biogeography of Alligatoroidea. Journal of Vertebrate Paleontology, 19(supplement 2): 9-100. DOI: 10.1080/02724634.1999.10 011201

Brochu C.A. (2013) Phylogenetic relationships of Palaeogene ziphodont eusuchians and the status of Pristichampsus Gervais, 1853. Earth and Environmental Science Transactions of the 
Royal Society of Edinburgh, 103(3-4): 521-550. DOI: 10.1017/S1755691013000200

Buffrénil V. de. (1982) Morphogenesis of bone ornamentation in extant and extinct crocodilians. Zoomorphology, 99: 155-166.

Busack S.D. \& Pandya S. (2001) Geographic variation in Caiman crocodilus and Caiman yacare (Crocodylia, Alligatoridae): Systematics and legal implications. Herpetologica, 57: 294-312.

Carvalho A.L. (1951) Jacarés do Brasil. Arquivos Do Museu Nacional, 43: 127-153.

Castro M.C., Montefeltro F.C. \& Langer M.C. (2014) The Quaternary vertebrate fauna of the limestone cave Gruta do Ioiô, northeastern Brazil. Quaternary International, 352(C): 164-175. DOI: 10.1016/j.quaint.2014.06.038

Chen I.H., Yang W. \& Meyers M.A. (2014) Alligator osteoderms : Mechanical behavior and hierarchical structure. Materials Science \& Engineering C, 35: 441-448. DOI: 10.1016/j.ms ec.2013.11.024

Cidade G.M. \& Hsiou A.S. (2018) The crocodylomorph fauna of the cenozoic of South America and its evolutionary history: A review Journal of South American Earth Sciences The crocodylomorph fauna of the Cenozoic of South America and its evolutionary history : a review. Journal of South American Earth Sciences, 90: 392-411. DOI: 10.1016/j.jsames.2018.12 .026

Clarac F., Buffrénil V. De, Cubo J. \& Quilhac A. (2018) Vascularization in Ornamented Osteoderms: Physiological Implications in Ectothermy and Amphibious Lifestyle in the Crocodylomorphs? The Anatomical Record, 183: 175-183. DOI: 10.1002/ar.23695

de Melo França L., Fortier D.C., Bocchiglieri A., Dantas M.A.T., Liparini A., Cherkinsky A. \& de Souza Ribeiro A. (2014) Radiocarbon dating and stable isotopes analyses of Caiman latirostris (Daudin, 1801) (Crocodylia, Alligatoridae) from the late Pleistocene of Northeastern Brazil, with comments on spatial distribution of the species. Quaternary International, 352(C): 159_ 163. DOI: 10.1016/j.quaint.2014.06.046

Dubansky B.H. (2012) The functional morphology of the intermandibulo-cervical envelope of the American alligator (Alligator mississippiensis). Doctoral thesis, Department of Biological Sciences, Louisiana State University and Agricultural and Mechanical College.

Dubansky B.H. \& Dubansky B.D. (2018) Natural Development of Dermal Ectopic Bone in the American Alligator (Alligator mississippiensis) Resembles Heterotopic Ossification Disorders in Humans. The Anatomical Record, 301: 56-76. DOI: 10.1002/ar.23682

Filho W.S., Karmann I., Boggiani P.C., Petri S., De Souza Cristalli P. \& Utida G. (2009) A deposição de tufas quaternárias no estado de Mato Grosso do Sul: Proposta de definição da Formação Serra da Bodoquena. Geologia USP - Serie Cientifica, 9(3): 47-60. DOI: 10.5327/Z1519-874X2009000300003

Fortier D.C. \& Rincón A.D. (2013) Pleistocene crocodylians from Venezuela, and the description of a new species of Caiman. Quaternary International, 305: 141-148. DOI: 10.1016/j.quaint.2012.03.018

Fortier D.C., Vilaboim L. \& Vasconcelos A. (2010) Primeiro registro de Caimaninae para o Quaternário do Estado da Bahia (p. 13-14). In: Riff D. (Org.). Paleo Minas. Livro de Resumos. Uberlândia: UFU.

Gmelin J.F. (1789) Pars III, Caroli a Linné, Systema Naturae. Leipzig: Forgotten Books, Germany, $492 \mathrm{p}$.

Godoi H.O. (2001) Programa Levantamentos Geológicos Básicos do Brasil, Aquidauana-MS. Folha SF. 21-X-A, Escala 1:250.000. Brasília: CPRM.

Gray J.E. (1844) Catalogue of the Tortoises, Crocodiles, and Amphisbaenians, in the Collection of the British Museum. London: British Museum (Natural History), Department of Zoology. 80 p.

Guidon N., Guérin C., Faure M., Felice G.D., Cristiane B. \& Ignácio E. (2009) Toca das Moendas, Piauí-Brasil, primeiros resultados das escavações arqueológicas. Fumdhamentos, 8: 70-85.

Hill R.V. (2010) Osteoderms of Simosuchus clarki (Crocodyliformes: Notosuchia) from the Late Cretaceous of Madagascar. Journal of Vertebrate Paleontology, 30(supplement 1): 154-176. DOI: $10.1080 / 02724634.2010 .518110$ 
Hirooka S.S. (2003) As cavernas do Bauxi como detentoras de informações do período Pleistoceno (p. 204-205). In: Simpósio de Geologia do Centro Oeste. Boletim de resumos. Volume 1. Cuiabá: UFMT.

Holz M. \& Simões M.G. (2002) Elementos fundamentais de tafonomia. Porto Alegre: UFRGS. 232 p.

Hsiou A.S. \& Fortier D.C. (2007) Primeiro registro de Caiman (Crocodylia, Alligatoridae) para o Pleistoceno do Estado do Rio Grande do Sul, Brasil. Gaea, 3(1): 37-44.

Lydekker R. (1888) Catalogue of fossil Reptilia and Amphibia. London: Trustees. 309 p.

Magnusson W.E. (1992) Paleosuchus palpebrosus (p. 554.1-554.2). In: King F.W. \& Brisbin Jr. I.L (Edits). Catalogue of American Amphibians and Reptiles. Manaus: Society for the Study of Amphibians and Reptiles.

Mapa político América do Sul (2019) Wikimedia Commons, the free media repositor: Mapa político da América do Sul. Disponível em: https://commons.wikimedia.org/wiki/File:Mapa_po 1\%C3\%ADti co_Am\%C3\%A9rica_do_Sul.svg (Acessado em: 16/04/19). Atribuição: High source [CC BY-SA 4.0 (https://creativecommons.org/licenses/by-sa/4.0)].

Marinho T.S., Porpino K. \& Santos M.F.C.F. (2005) Ocorrência de Caiman latirostris Daudin, 1802 (Crocodylia: Alligatoridae) no Quaternário do Rio Grande do Norte, Brasil (p. 163). In: Kellner A.W., Henriques D.D.R. \& Rodrigues T. (Orgs). II Congresso Latino-Americano de Paleontologia de Vertebrados (Resumos). Rio de Janeiro: Museu nacional/UFRJ/FAPERJ.

Oliveira A.M. (2013) Paleofauna de vertebrados, com ênfase em répteis e mamíferos, dos depósitos quaternários da região da Serra da Bodoquena, Mato Grosso do Sul, Brasil. Dissertação de Mestrado, Programa de Pós-Graduação em Geologia Regional. Universidade Estadual Paulista, Rio Claro, São Paulo.

Oliveira A.M. de \& Cordeiro L.M. (2017) Novas ocorrências de Scelidotheriinae (Mylodontidae) em cavernas da Serra da Bodoquena (Mato Grosso do Sul, Brasil). Espelo-Tema, 28(2): 125134.

Oliveira A.M.de, Becker-kerber B., Cordeiro L.M., Borghezan R., Avilla L.S. \& Santos C.M.D. (2017) Quaternary mammals from central Brazil (Serra da Bodoquena, Mato Grosso do Sul) and comments on paleobiogeography and paleoenviroments. Revista Brasileira de Paleontologia, 20(1): 31-44. DOI: 10.4072/rbp.2017.1.03

Pansani T.R., Oliveira A.M. de \& Pacheco M.L.A.F. (2016) Nova ocorrência de megafauna plesitocênica em Mato Grosso do Sul. Revista Do Instituto Geológico, 37(2): 73-85. DOI: 10.5935/0100-929X.20160010

Patterson B. (1936) Caiman latirostris from the Pleistocene of Argentina, and a summary of South American Cenozoic Crocodilia. Herpetologica, 1(2): 43-54.

Pereira M.C.B., Mendes C.A.B., Grehs S.A., Barreto S.R., Becker M., Langer M.B.R. \& Dias F.A. (2004) Bacia Hidrográfica do Rio Miranda: Estado da Arte. Campo Grande: UCDB. 177 p.

Porpino K., Marinho T.S. \& Santos M.F.C.F. (2004) Ocorrência de Crocodiliformes no Quaternário do Lajedo de Soledade, Apodi (RN) (p. 49-56). In: Netto R.G. (Org.). Boletim da Sociedade Brasileira de Paleontologia. Rio de Janeiro.

Richardson K.G., Webb G.J.W. \& Manolis S.C. (2002) Crocodyles: inside out. A guide to the crocodylians and their functional morphology. Australia: Surrey Beatty and Sons. 172 p.

Romer A.S. (1997) Osteology of the reptiles (Reprint Ed). Berlin: Malabar Krieger Publishing Company. 800 p.

Salisbury S.W. \& Frey E. (2001) A biomechanical transformation model for the evolution of semi-spheroidal articulations between adjoining vertebral bodies in crocodilians (p. 85-134). In: Grigg G.C., Seebacher F. \& Franklin C.E. (Orgs). Conference on Crocodilian Biology and Evolution, 1998. Australia: Surrey Beatty \& Sons, St Lucia.

Salisbury S.W., Molnar R.E., Frey E. \& Willis P.M.A. (2006) The origin of modern crocodyliforms: new evidence from the Cretaceous of Australia. Proceedings of the Royal Society B: Biological Sciences, 273: 2439-2448. DOI: 10.1098/rspb.2006.3613

Salles L.O., Cartelle C., Guedes P.G., Boggiani P.C., Janoo A. \& Russo C.A.M. (2006) Quaternary mammals from Serra da Bodoquena, Mato Grosso do Sul, Brazil. Boletim Do Museu 
Nacional, (521): 1-12.

Scheffler M.S., Kashimoto E.M. \& Oliveira A.M. (2010) Revisão sobre a Paleontologia no Estado do Mato Grosso do Sul: fósseis e afloramentos descritos. Brazilian Geographical Journal: Geosciences and Humanities Research Medium, 1: 65-99.

Tucker A.D. (1997) Validation of skeletochronology to determine age of freshwater crocodiles (Crocodylus johnstoni). Marine Freshwater Research, 48: 343-351.

Turcq B., Suguio K., Soubies F., Servant M. \& Pressinotti M.M.N. (1987) Alguns terraços fluviais do Sudeste e do Centro-oeste brasileiro datados por radiocarbono: possíveis significados paleoclimáticos (p. 379-392). In: Correa I.R.S. (Org.). Anais do Congresso da Associação Brasileira de Estudos do Quaternário. Porto Alegre: ABEQUA.

Vitt L.J. \& Caldwell J.P. (2009) Herpetology: an introductory biology of amphibians and reptiles. $3^{\circ}$ edition. UK/USA: Academic Press. 713 p.

Wermuth H. \& Fuchs K. (1978) Wermuth, Heinz, and Karlheinz Fuchs. Bestimmen von Krokodilen und ihrer Häute: e. Anleitung zum Identifizieren d. Art-u. Rassen-Zugehörigkeit d. Krokodile für Behörden (im Zusammenhang mit d. Artenschutz-Übereinkommen von Washington), für Reptilleder-Indu. Fischer; 1. Aufl edition. 99 p.

Salisbury W.S. \& Frey E. (2001) A biomechanical transformation model for the evolution of semi-spheroidal articulations between adjoining vertebral bodies in crocodilians (p. 85-154). In: Grigg G.C., Seebacher F. \& Franklin C.E. (Eds). Crocodilian Biology and Evolution. Chipping Norton: Surrey Beatty and Sons. 446 p. 